\title{
New antimicrobial prescribing guidelines for dentists
}

The Faculty of General Dental Practice UK (FGDP) and the Faculty of Dental Surgery of the Royal College of Surgeons of England (FDS) have published the third edition of Antimicrobial Prescribing in Dentistry - Good Practice Guidelines.

Developed by an intercollegiate working group led by Dr Nick Palmer, it updates FGDP's well-known Antimicrobial Prescribing for General Dental Practitioners guidance to reflect changes in the evidence base since the previous edition, and covers a much wider range of conditions.

Its scope has been extended to include management of oral and dental infections by all prescribers, not only general dental practitioners but those working in secondary dental care (including trainees), specialists (including oral and maxillofacial surgeons), and those involved in dental education and research. Its recommendations are also now appropriate for all dental patients, including adults, children, the elderly and those with special needs treated in the primary and secondary care setting.

Inappropriate prescribing of antimicrobials can exacerbate the problem of antimicrobial resistance, which leads to antibiotics no longer being effective in treating even simple infections. There are serious consequences for everyone, but particularly those undergoing major surgery, chemotherapy, organ or stem cell transplants. Every year, 25,000 people

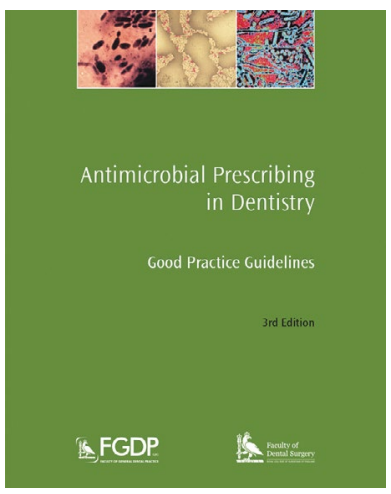

across Europe, and 700,000 worldwide, die from antibiotic-resistant infections, and the UK government predicts the annual global toll could be 10 million by 2050 .

Prudent prescribing of antimicrobials can slow down the further development of antimicrobial resistance, and all healthcare prescribers play a vital role. Dentists prescribe an estimated $10 \%$ of all oral antimicrobials prescribed in England, and are required by legislation to ensure appropriate use of antimicrobials.

Published following consultation with a range of specialist societies and national dental organisations, the new guidelines are available free of charge in PDF format at https://www.fgdp.org.uk/guidance-standards and https://www.rcseng.ac.uk/dentalfaculties/fds/publications-guidelines/clinicalguidelines, and print copies can be purchased at https://www.fgdp.org.uk/shop.

\section{Three new ambassadors for CGDent}

The College of General Dentistry - the first independent college for primary care dentistry in the UK - has appointed three new Ambassadors: Professor Richard

Horton, Dr Govin Murugachandran and Professor Jonathan Cowpe.

College Ambassadors support the engagement of the College in general healthcare and society and promote its influence in the interests of patients and the public, building trust and confidence in the dental profession.

Chair of the College Board of Trustees, Professor Nairn Wilson, said: 'I am delighted that Professors Horton and Cowpe and Dr Murugachandran have joined the College's exceptional team of Ambassadors. Collectively, they have a huge range of highly relevant expertise and knowledge, with opportunity to greatly increase awareness of the College and its importance. The College looks forward to having the support and encouragement of its new Ambassadors in working towards realising the goals of excellence in oral healthcare provision and new leadership and support for all dental healthcare professionals'

\section{Dental organisations investigate discrimination}

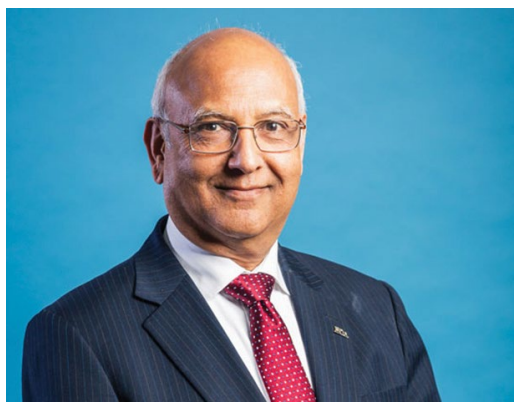

The British Dental Association, Faculty of General Dental Practice UK and College of General Dentistry have joined forces to conduct a survey into discrimination in dentistry.

The survey is aimed at all dentists who have ever practised dentistry in the UK, and at dental students studying in the UK, and asks about their morale and career satisfaction as well as any experiences they may have had of being treated unfairly whilst working or studying.

Feedback from the survey will be anonymised, and the data and experiences captured will be used to identify and raise awareness of the ways in which discrimination is experienced in dentistry.

In recent months, the three organisations have convened a programme board to promote joint working on issues of equality, diversity and inclusion (EDI) in dentistry, as well as a joint working group to investigate racial inequality in particular. The survey results will help these groups take forward evidence-based actions to address the underlying issues, and to promote greater understanding in the profession of the experiences and concerns of their fellow dentists.

The survey is available at https://www. smartsurvey.co.uk/s/racismindentistry/.

Russ Ladwa, President of the BDA (pictured), said: 'Discrimination is unfortunately still a feature of our society, and dentistry is far from immune, but this survey is an important step towards understanding and addressing the issue in our profession, and I encourage all dentists to complete it? 